\title{
Introduction: biomarkers in heart failure
}

\author{
Kirkwood F. Adams
}

Published online: 7 May 2009

(C) Springer Science+Business Media, LLC 2009

Practitioners have long known that the clinical manifestations of cardiovascular disease, exemplified by signs and symptoms, may be nonspecific and confusing during the diagnostic process. Worse, these classical clinical indicators do not fully reflect the extent of the underlying pathophysiological process and may give an incomplete picture of prognosis. In addition, the treatment of cardiovascular disease has become increasingly complex, with polypharmacy, the rule and novel devices often of benefit, but standard clinical assessment often does not indicate what combination of therapy should be used or adequately reflect the response to treatment. Finally, signs and symptoms typically emerge late in the natural history of these disorders, delaying their identification until after preventative measures and early treatments could have reduced disease burden. These considerations point to the limitations of standard clinical assessment for the diagnosis, prognostic stratification, and monitoring of therapeutic response in cardiovascular disease and indicate more sophisticated characterization of the clinical phenotype is needed.

Biomarkers are emerging as one powerful adjunct to standard clinical assessment in a variety of cardiovascular disorders including heart failure. Biomarkers function as physiological measurements that may be molecular in nature (specific hormone concentration, genotype, or protein expression) or reflect macroscopic organ function (estimated pulmonary artery pressure or ambulatory blood

\footnotetext{
K. F. Adams ( $\square)$

Departments of Medicine and Radiology, School of Medicine, UNC Heart Failure Program, University of North Carolina at Chapel Hill, 730 Martin Luther King Jr Blvd, Suite 207, Chapel Hill, NC 27514, USA

e-mail: kfa@med.unc.edu
}

pressure) [1]. The ability of biomarkers to provide more accurate and sensitive detection and characterization of cardiovascular disease than clinical assessment alone is becoming more widely accepted [2]. Ongoing investigation suggest that these physiological measurements, which may be defined as biological parameters subject to quantitative determination, are of significant clinical value as they appear to accurately reflect the extent of disease physiology, prognosis, and likely response to therapy.

In this special edition of Heart Failure Reviews, composed by an international group of experts, various aspects of the current clinical utility of biomarkers in heart failure are presented along with an in depth discussion of many novel aspects and emerging concepts related to biomarkers in this major cardiovascular syndrome. Molecular biomarkers that reflect critical systems in the pathophysiology of heart failure (fibrosis or inflammation) and important markers of disease activity in this syndrome (natriuretic peptides) are emphasized in the review. As expected, several papers focus on natriuretic peptides, which represent a highly successful application of biomarkers in heart failure, and whose role continues to be refined and expanded [3]. Chiong, $\mathrm{Pu}$, and co-authors provide comprehensive and detailed reviews of how natriuretic peptides have enhanced diagnosis and prognostic stratification in both acute and chronic heart failure and how they vary in other clinical subsets of heart failure and other important disease states.

As illustrated by the remainder of the papers, many new markers and strategies for the utilization of biomarkers as physiological probes and aids in management have been developed these approaches that build upon the solid foundation established by the natriuretic peptides. Rocchiccioli, McMurray, and Dominiczak begin the issue with a comprehensive review of biomarkers currently 
established or under investigation for clinical use in heart failure. Bass and colleagues provide a comprehensive overview of cardiac troponins, rapidly emerging along with natriuretic peptides as useful adjuncts in the prognostic stratification of acute and chronic heart failure and the assessment of cardiac toxicity. Zannad and co-authors provide an excellent presentation of markers of fibrosis, an aspect of cardiac remodeling that remains insufficiently understood and accounted for in heart failure. The paper of Bozkurt et al. focuses on inflammatory molecules and illustrates the potential and need for ongoing investigation of the clinical utility of these molecules. Allen and Felker discuss the concept of a multimarker approach to diagnosis and prognosis in heart failure, which will be an increasingly important strategy as multiple markers with clinical information independent of natriuretic peptides emerge.

These articles set the stage for the final two papers, one on biomarker guided therapy and the other on systems biology in heart failure, which delineate two major areas of new research activity with potentially profound implications concerning biomarkers in heart failure. Using biomarkers to guide therapy represents a way to fulfill the promise of biomarkers to promote personalized medicine and revolutionize strategies to optimize therapy in cardiovascular disease [4]. As outlined in the paper exploring guided therapy, ample pilot data are available to support the conduct of a definitive outcomes study to prove this highly generalizable approach to clinical care. Definitive, prospective and properly designed, conducted, and monitored large-scale trials are essential to move this critical field of medicine forward.

Systems biology represents a very important contemporary area of biological science and research $[5,6]$. This discipline has evolved into a much more rigorous scientific pursuit as a result of dramatic progress in many disciplines including not only molecular biology, with characterization of the complex metabolic and regulatory networks that make life possible and account for disease, but also advances in bioinformatics and clinical phenotyping [7-9]. Systems biology makes heavy use of various omics: genomics, transcriptomics, and proteomics as a partial list, in characterizing biology. Collectively referred to as biomedomics in this review, these individual disciplines have all seen significant recent advances, which make their application to human disease much more likely to provide valuable insights. In its full realization, systems biology is analogous to the Theory of Everything in physics, a triumph not near realization, which has caused some to speculate that its application is too exploratory and its ultimate goals unobtainable [10,11]. However, advances in many biomedomic fields now make plausible research into the comprehensive application of this methodology to cardiovascular diseases like heart failure [12]. This review emphasizes key aspects of the systems biology perspective that can be systematically applied to the study of heart failure today.

In closing, the clinical application of biomarkers in medicine represents an attempt to shift traditional paradigms used for centuries in the evaluation and management of patients with cardiovascular disease. This review issue illustrates both the progress and the research task that remains to bring this aspect of personalized medicine to it full potential.

\section{References}

1. Tang WH, Francis GS, Morrow DA, Newby LK, Cannon CP, Jesse RL, Storrow AB, Christenson RH, Apple FS, Rayklide J, Wu AH, National Academy of Clinical Biochemistry Laboratory Medicine (2007) National Academy of Clinical Biochemistry Laboratory Medicine practice guidelines: clinical utilization of cardiac biomarker testing in heart failure. Circulation 116:e99e109. doi:10.1161/CIRCULATIONAHA.107.185267

2. Vasan RS (2006) Biomarkers of cardiovascular disease: molecular basis and practical considerations. Circulation 113:23352362. doi:10.1161/CIRCULATIONAHA.104.482570

3. Maisel A, Mueller C, Adams KF Jr, Anker SD, Aspromonte N, Cleland JGF, Cohen-Solal A, Dahlstrom U, DeMaria A, Di Somma S, Filippatos GS, Fonarow GC, Jourdain P, Komajda M, Liu PP, McDonagh T, McDonald K, Mebazaa A, Nieminen MS, Peacock F, Tubaro M, Valle R, Vanderhyden M, Yancy CW, Zannad F, Braunwald E (2008) State of the art: using natriuretic peptide levels in clinical practice. Eur J Heart Fail 10:824-839. doi:10.1016/j.ejheart.2008.07.014

4. Troughton RW, Frampton CM, Yandle TG, Espiner EA, Nicholls MG, Richards AM (2000) Treatment of heart failure guided by plasma amino terminal brain natriuretic peptide (N-BNP) concentrations. Lancet 355:1126-1130. doi:10.1016/S0140-6736(00) 02060-2

5. Ideker T, Galitski T, Hood L (2001) A new approach to decoding life: systems biology. Annu Rev Genomics Hum Genet 2:343372. doi:10.1146/annurev.genom.2.1.343

6. Hartwell LH, Hopfield JJ, Leibler S, Murray AW (1999) From molecular to modular cell biology. Nature 402(6761, Suppl):C47-C52. doi:10.1038/35011540

7. Watson JD, Crick FH (1953) Molecular structure of nucleic acids; a structure for deoxyribose nucleic acid. Nature 171:737738. doi:10.1038/171737a0

8. Heidecker B, Hare JM (2007) The use of transcriptomic biomarkers for personalized medicine. Heart Fail Rev 12:1-11. doi: 10.1007/s10741-007-9004-7

9. Arab S, Gramolini AO, Ping P, Kislinger T, Stanley B, van Eyk J, Ouzounian M, MacLennan DH, Emili A, Liu PP (2006) Cardiovascular proteomics: tools to develop novel biomarkers and potential applications. J Am Coll Cardiol 48:1733-1741. doi: 10.1016/j.jacc.2006.06.063

10. Barrow JD (2005) New theories of everything. Oxford University Press, Inc, New York, NY

11. Lisi AG (2007) An exceptionally simple theory of everything. arXiv: 0711.0770

12. Feero WG, Guttmacher AE, Collins FC (2008) The genome gets personal almost. J Am Med Assoc 299:1351-1352. doi:10.1001/ jama.299.11.1351 